\title{
RECONSIDERATION OF THE GALVANOSTATIC DOUBLE-PULSE METHOD
}

D. J. KOOIJMAN AND J. H. SLUYTERS

Laborafory of Analytical Cfemistry. State Uniwersity. Utrecht (Netherlands)

(Received February 3rd, I966)

Fast electrode processes can be studied only by relaxation methods. In these methods, the electrode processes should be perturbed with signals of high frequency or short time in order to minimize concentration polarization.

One of the limitations encountered in the study of electrode kinetics with the galvanostatic single-pulse method is the influence of the double-layer capacitance at short pulse lengths. To remove this limitation, GERIScher AND KRAUSE' developed the galvanostatic double-pulse method. The first pulse which is of higher amplitude, $i_{1}$, than the second pulse, primarily charges the double-layer capacitance to the overvoltage required by the current density, $i_{2}$, of the second pulse; $i_{\text {.e. }}$, the overvoltage-time curve should start with a horizontal tangent at the beginning of the second pulse. Thus the current, passing the cell, is entircly faradaic at that moment. The value $i_{1} / i_{2}$ must be found by trial and error.

A mathematical analysis of the method, limited to cell responses of small amplitude, has been given by DELAHdy et al.?. They correct for the concentration polarization occurring within the time, $t_{1}$, of the first pulse. However, they do not consider the errors caused by the experimental difficulty of observing whether or not the cell response starts with a horizontal tangent at time $t_{1}$. In the present paper we shall show tobat this difficulty seriously limits the double-pulse method.

THEORY

According to DeLAHAy et al-2, the voltage-time relation for the response of an electrode process, that is perturbed by a double-pulse current (Fig. $I$ ), is:

$$
\begin{aligned}
\eta(t) & =\frac{i_{1}}{C_{d}(\gamma-\beta)}\left[\gamma / \beta^{2}\left(\exp \beta^{2} t \operatorname{erfc} \beta \sqrt{t}+2 \beta \sqrt{\frac{t}{\pi}}-\mathrm{I}\right)\right. \\
& \left.-\beta / \gamma^{2}\left(\exp \gamma^{2} t \operatorname{erfc} \gamma l^{/ t}+2 \gamma \sqrt{\frac{t}{\pi}}-\mathrm{I}\right)\right] \\
& +\frac{i_{2}-i_{1}}{C_{d}(\gamma-\bar{\beta})}\left[\gamma / \beta^{2}\left(\exp \beta^{2}\left(t-t_{1}\right) \operatorname{erfc} \beta \sqrt{t-t_{\mathrm{L}}}+2 \beta \sqrt{\frac{t-t_{1}}{\pi}}-I\right)\right. \\
& \left.-\gamma / \beta^{2}\left(\exp \gamma^{2}\left(t-t_{1}\right) \operatorname{erfc} \gamma \sqrt{t-t_{1}}+2 \gamma \sqrt{\frac{t-t_{\mathrm{L}}}{\pi}}-I\right)\right]
\end{aligned}
$$

and if $\left(\frac{d \eta}{d t}\right)_{t-t}=0$, then

J. Eledroanal. Chem., ${ }_{3}\left(\mathrm{I}_{967}\right) \mathrm{I}_{52-156}$ 


$$
\frac{i i_{2}}{i_{1}}=I-\frac{I}{\gamma-\beta}\left[\gamma \exp \beta=t_{1} \operatorname{erfc} \beta \sqrt{t_{1}}-\beta \exp \gamma^{2} t_{1} \operatorname{erfc} \gamma \sqrt{t_{1}}\right]
$$

in which

$$
\beta+\gamma=\frac{i_{0}}{n F}\left(\frac{I}{C_{0}^{* *} \sqrt{D_{0}}}+\frac{I}{C_{\mathrm{R}}^{*} \sqrt{D_{\mathrm{R}}}}\right) \text { and } \beta \gamma=\frac{n F i_{0}}{R C_{a}} \text {. }
$$

The interpretation of equ. (I) is difficult. Therefore we shall simplify it assuming that $t_{\overline{1}} \rightarrow 0$ and $i_{1} t_{1} / C_{a} \rightarrow \eta_{0}$. Equation ( $\mathrm{I}$ ) then reduces to:

$$
\begin{aligned}
\eta(t)= & \frac{\eta_{o}}{\gamma-\beta}\left[\gamma \exp \beta^{2} t \operatorname{exfc} \beta \sqrt{t}-\beta \exp \gamma^{2 t} \operatorname{erfc} \gamma \sqrt{t}\right] \\
& +\frac{i_{2}}{C_{d}(\gamma-\beta)}\left[\gamma / \beta^{2}\left(\exp \beta^{2} t \operatorname{erfc} \beta \sqrt{t}+2 \beta \sqrt{\frac{t}{\pi}}-\mathrm{I}\right)\right. \\
& \left.-\beta / \gamma^{2}\left(\exp \gamma^{2} t \operatorname{erfc} \gamma \sqrt{\mathrm{t}}+2 \gamma \sqrt{\frac{t}{\pi}}-\mathrm{I}\right)\right]
\end{aligned}
$$

The condition, $(\mathrm{d} \eta / \mathrm{d} t)_{t=0}=0$, yields

$$
i_{2}=\beta \gamma C_{a} \eta_{0}
$$

If $i_{2}=A \beta_{\gamma} C_{a \eta}$ o, the curve will not start with a horizontal tangent, and for $A<\mathrm{I}$ the curve will exhibit a minimum at a time $t_{m}$, see Fig. 2 . Obviously $i_{2}$ should be adjusted so that this minimum has just disappeared. This means that $\Delta \eta$ must be distinct for a small deviation of $i_{2}$ from the correct value.
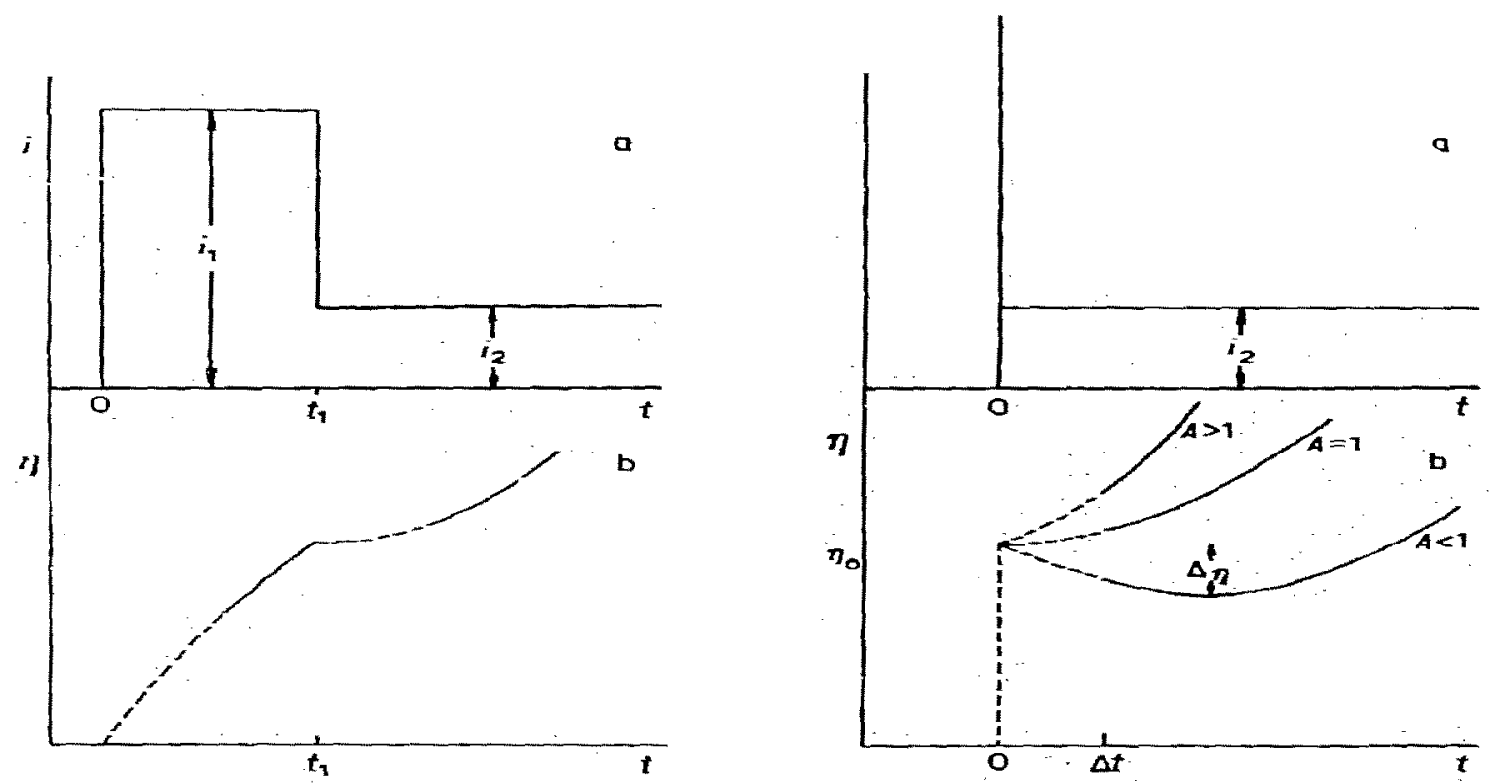

Fig. I. (a), Shape of a double-pulse current; (b); cell response having a horizuntal Langent at ume $t_{1}$. The dashed parts of the curve indicate that in these regions the response is obscured by transients.

Fig. 2: (a), Double-pulse current with an infinitely short first-pulse length: (b): cell response having $a$ horizontal tangent at time $t=0$. The dashed part of the curve indicates the region, $\Delta t$, which is obscured by transients. 
The values $t_{m}$ and $\Delta \eta$ can be obtained by expansionin series of the exponential and error functions in eqn. (3) for small values of $t$ :

$$
\begin{aligned}
& \eta(t)=\eta_{0}\left[\mathrm{I}-(\mathrm{I}-A) \beta \gamma t+\frac{4}{3 \sqrt{\pi}} \beta \gamma(\beta+\gamma) t / t\right] \\
& \frac{\mathrm{d} \eta}{\mathrm{d} t}=\mathrm{o} \quad \text { yields } t_{n}=\frac{\pi}{4}\left(\frac{\mathrm{I}-A}{\beta+\gamma}\right)^{2}
\end{aligned}
$$

and

$$
\frac{\Delta \eta}{\eta_{0}}=-\frac{\pi}{\mathrm{I}^{2}}(\mathrm{I}-A)^{3} \frac{\beta \gamma}{(\beta+\gamma)^{2}}
$$

These equations are valid onIy if $\beta \gamma^{t}<I$ and $(\beta+\gamma) t^{ \pm}<x$. These conditions will be checked afterwards.

From Fig. 2 it can be seen that, in order to observe a minimum in the cell response, $\Delta \eta / r_{j}$ must have a finite value. Moreover $t_{m}$ must be larger than $\Delta t$, the fime during which the cell response is obscured by transients.

\section{THE VALUES OF $\Delta t$ AND $\Delta \eta$}

Even with good electronic design, transients, due to finite time constants of cables and the cell in the circuit, will be observed at the initial part of the potentialtime curve. We have found experimentally that these transients have been damped only after $0.4 \mu \mathrm{sec}$. Consequently, $t_{m}$ must be larger than $0.4 \mu \mathrm{sec}$. Not only transients will contribute to $\Delta t$, but also the rise and decay times of the pulse-generators and the response time of the oscilloscope recording the overvoltage-time curve. If the ohmic drop in the cell is compensated by means of a bridge circuit of DELARAY ${ }^{3}$, the value of $\Delta t$ will be generally larger than I $\mu \mathrm{sec}$, as a differential oscilloscope with relatively poor rise time must be uscd. Elsewheres we have described a set-up for the compensaticn of the ohmic drop in which an asymmetric fast response oscilloscope can be used. In that case $\Delta t$ will be about $0.4 \mu \mathrm{sec}$.

The value of $\Delta \eta$ first depends on the line-width of the trace on the screen of the oscilloscope, generally $\Delta \eta / \eta \geqslant 0.0 x$. However, if high exchange current densities are measured, the compensation of the ohmic drop will be more important. If $e_{-g}$. the miscompensation of the ohmic drop for the second pulse is $0.05 \mathrm{mV}$, the first part of the curve, from $o$ to $t_{1}$, will be shifted an amount $\left(i_{1}-i_{2}\right) / i_{2} \cdot 0.05 \mathrm{mV}$ with respect to the second part of the curve, $t>t_{1}$. As a result, $\Delta \eta$ must be larger than o.or $\eta$ in order to observe a minimum in the cell response. If the influence of a miscompensation $0 \underline{m}$ the ohmic drop is also taken into account, a value of $\Delta \eta / \eta \mathbf{\eta} \geqslant 0.0 z$ seems reasonable.

SCOPE OF THE DOUBLE-PULSE METHOD

Inserting $t_{m} \geqslant 0.4 \mu \mathrm{sec}$ and $\Delta \eta / \eta_{0} \geqslant 0.02$ in eqns. (6a) and (6b), one obtains: for $\mathrm{A}=0.9: \quad \beta+\gamma \leqslant 140$ and $\beta \gamma \geqslant 76(\beta+\gamma)^{2}$

$$
\left.\begin{array}{lll}
\mathrm{A}=0.8: \beta+\gamma \leqslant 280 & \beta y \geqslant 9.6(\beta+\gamma)^{2} \\
\mathrm{~A}=0.5: \beta+\gamma \leqslant 700 & \beta \gamma \geqslant 0.6 \mathrm{x}(\beta+\gamma)^{2}
\end{array}\right\}
$$

J. Electroanal. Cheirz., $13(1967)$ 152-156 
Taking the following reasonable values: $n=2, T=300^{\circ} \mathrm{K}, D_{\mathrm{ox}}=D_{\mathbf{B}}=\mathrm{IO}^{-5}$ $\mathrm{cm}^{2} \mathrm{sec}^{-1}, C_{a}=25 \cdot \mathbf{x}^{-6} \mathrm{Fcm}^{-2}$ and $C_{\mathrm{ox}^{*}}=C_{\mathrm{R}^{*}}=C^{*}$, one can calculate the limits of $k_{s h}$ and $i_{0}$, which should not be exceeded if accuracies of 10,20 and $50 \%$ are required, respectively, see Table I.

TABLE 1

\begin{tabular}{|c|c|c|c|}
\hline$A$ & $\begin{array}{l}C^{*} \\
\left(m o l e c m^{-2}\right)\end{array}$ & ${ }^{i_{0}}\left(A \mathrm{~cm}^{-3}\right)$ & $\begin{array}{l}K_{a n} \\
\left(c m \sec ^{-1}\right)\end{array}$ \\
\hline 0.9 & $\begin{array}{l}10^{-7} \\
5 \cdot 10^{-7} \\
2-5 \cdot 10^{-6} \\
\text { Io- } 10^{-6} \text { and higher }\end{array}$ & $\begin{array}{l}\leqslant 44 \cdot 10^{-6} \\
\leqslant 1.1-10^{-3} \\
\leqslant 27 \cdot 10^{-9}\end{array}$ & $\begin{array}{l}\leqslant 22-10^{-3}(10 \%) \\
\leqslant 1.1-10^{-2} \\
\leqslant 5 \cdot 4-10^{-2} \\
\leqslant 2.2-10^{-1}\end{array}$ \\
\hline 0.8 & $\begin{array}{l}10^{-2} \\
5 \cdot 10^{-7} \\
2.5^{-10^{-6}} \text { and higher }\end{array}$ & $\begin{array}{l}\leqslant 350-10^{-0} \\
\leqslant 8.8 \cdot 10^{-3} \\
-\end{array}$ & $\begin{array}{l}\leqslant 1.7-10^{-2}(20 \%) \\
\leqslant 8.8-10^{-2} \\
\leqslant 4-4-10^{-1}\end{array}$ \\
\hline 0.5 & $\begin{array}{l}\text { 10-7 } \\
4^{-10^{-7}} \text { and higher }\end{array}$ & $\leqslant 5.2 \cdot \mathrm{IO}^{-3}$ & $\begin{array}{l}\leqslant 0.26 \\
\leqslant 1\end{array}$ \\
\hline
\end{tabular}

It should be noted that the error in the measured $i_{0}$ or $k_{5 h}$ is a systematic one. The measured value for the exchange current density or rate constant is always too low.

A numerical examination* of eqn. (I) revealed that even if $t_{1}>0$, the same limits, as tabulated above, are valid provided that the correction for concentration polarization, occurring within time $t_{1}$, is performed with sufficient accuracy. This may be difficult if the rate constant, $k_{\mathrm{sh}}$, is large, as the extrapolation procedure proposed by Delafay ${ }^{2}$ et al, is not always allowed.

DETERMINATION OF THE EXCHANGE CURRENT DENSITY FOR THE $\mathrm{Hg} / \mathrm{Hg}^{2+}$ ELECTRODE REACTION IN I $M \mathrm{HClO}_{4}$

The $\mathrm{Hg} / \mathrm{Hg}^{2}$ r electrode has been investigated by means of several relaxation methods and by different authors. IMAI AND DELAHAY ${ }^{5}$ reported for I $\operatorname{mM} \mathrm{Hg}_{2}{ }^{2+}$, an exchange current of I2 $\mathrm{Acm}^{-2}$, measured with the faradaic rectification method. SLUYTERS-REHBACH AND SLUYTERS ${ }^{6}$ conclude from a complex plane analysis that $i_{0}$ must be larger than $45^{\circ}$ iwA $\mathrm{cm}^{-2}$ for I mM $\mathrm{Hgg}^{2+}$. Birke and Roe ${ }^{7}$ measured with the galvanostatic single-pulse method an $i_{0}$ of about $350 \mathrm{~mA} \mathrm{~cm}^{-2}$. The following data are reported from the galvanostatic double-pulse method.

\begin{tabular}{|c|c|c|c|}
\hline \multirow[t]{2}{*}{$c+(m M)$} & \multicolumn{3}{|c|}{$i_{0}(m A c m-=)$} \\
\hline & Delahayz & Gerischer 1 & Ozer data \\
\hline 2 & 420 & 350 & 700 \\
\hline I & 250 & 200 & 400 \\
\hline 0.5 & 180 & 120 & 200 \\
\hline 0.2 & 75 & & 80 \\
\hline O.I & & & 35 \\
\hline
\end{tabular}

* Table of exp $x^{3}$ erfc $x$ are tabulated by Faddeyeva and Terent'ev'.

* These values are corrected for concentration polarizaliun occurring within the first pulse. 
The results obtained with the different relaxation methods are inconsistent. As regards the double-pulse method, it can be shown by means of eqn. ( 7 ) (in which, for $C_{d}$, the values published by SLUYTERS-REHBACH AND SLUYTERS ${ }^{8}$ are substituted) that the reported data contain errors larger than 50\%. Therefore one may conclude that the $\mathrm{Hg} / \mathrm{Hg}_{2}{ }^{2+}$ electrode reaction behaves reversibly with respect to the doublepulse method. The inconsistency between our data for $\mathrm{I} \mathrm{m} M$ and $2 \mathrm{~m} M \mathrm{Hg}^{2+}$ and those of DELAHAY AND GERIScher can be explained by assuming that their value of $\Delta t$ was larger than ours.

\section{CONCLUSIONS}

The galvanostatic double-pulse method has no advantage over the singlepulse method, as the experimental conditions to be fulfilled in order to measure high exchange current densities, canrot be realized. For the same reason, the values of $i_{0}$ for the $\mathrm{Hg} / \mathrm{Hg}_{2}{ }^{2+}$ electrode reaction measured with the double pulse method, published previously and in this paper, have no meaning.

\section{SUMHARY}

A critical examination is made of the voltage response of a cell when a doublepulse current is applied. The limitations of the double-pulse method for studying fast electrode reactions are considered and it is shown that the maximum value of the heterogeneous rate constant measnrable with this method is about $0.5 \mathrm{~cm} \mathrm{sec}^{-1}$. In this case the error will be $20 \%$.

\section{REFERENCES}

I H. Gerischer and M. Krause. Z. Physik. Chem. N.F., ic (1957) 264-

2 H. Matsuda, S. Ofa and P. Delahay, $J$. Am. Chem. Soc., 8 i (I959) 5077.

3 T. Berzisis and P. Delahay, $J$. Am. Chem. Soc., 77 (1955) 6448.

4 D. J. IKootj Man aid J. H. SiUrters, Electrochim. Acta, submitted.

5 H Imai and P. Delahay, $J$ - Phys. Chem., 66 (I962) 1 Io8.

$6 \mathrm{M}$. Sluyters-Rehbach and J. H. Sluyters, Rec. Trav. Chim., 83 (1964) 983.

7 R. L. Birke And D. K. RoE, Anal. Chem., 37 (1965) 45 r.

8 M. SluYters-Rehbach AxD J. H. SluYters, Rec. Trau. Chim., 83 (1964) 987.

9 V. N. FaDDEYeVA aNd N. M. TERENT'Ev, Tables of the probability integral for complex argitment, 1954: Engl- translation, Pergamon Press, Oxford, rg6i.

J. Electroanat. Chem., $13(1967) \times 52-156$ 